\title{
MicroRNA-340 Inhibits Esophageal Cancer Cell Growth and Invasion by Targeting Phosphoserine Aminotransferase 1
}

\author{
Shuo Yan ${ }^{a}$ Haosheng Jiang ${ }^{a}$ Shiming Fang ${ }^{a}$ Fang Yin ${ }^{a}$ Zhenlei Wang ${ }^{a}$ Yiping Jia ${ }^{a}$ \\ Xianjun Sun ${ }^{\mathrm{a}}$ Shaoqiu Wu ${ }^{\mathrm{a}}$ Tinghui Jiang ${ }^{\mathrm{a}}$ Aiwu Mao ${ }^{\mathrm{a}}$ \\ aDepartment of Interventional Radiology, Tongren Hospital, Shanghai Jiaotong University School of \\ Medicine, Shanghai, 'bepartment of Interventional Radiology, Tongren Hospital, Shanghai Jiaotong \\ University School of Medicine, Shanghai, PR China
}

\section{Key Words}

ESCC • PSAT1 • MiR-340 • Cell proliferation • Cell invasion

\begin{abstract}
Background/Aims: Emerging evidence indicates that microRNA (miR)-340 is downregulated in various human cancers, suggesting that it acts as a tumor suppressor. The aim of the present study was to evaluate the expression and role of miR-340 in human esophageal squamous cell carcinoma (ESCC). Methods: The expression of miR-340 was examined in 64 paired ESCC and adjacent non-tumor tissues by quantitative real time PCR. The effects of miR340 on ESCC cell proliferation and metastasis were examined by MTT and Matrigel invasion assays. Tumor growth was assessed by subcutaneous inoculation of cells into BALB/c nude mice. Targets of miR-340 were identified by bioinformatics and verified by luciferase reporter assays, quantitative real-time PCR, and western blotting. Results: MiR-340 was significantly downregulated in ESCC tumor tissues compared to adjacent non-tumor tissues and in ESCC cell lines compared to esophageal endothelial cells. Overexpression of miR-340 inhibited ESCC cell growth, colony formation, and invasion, and tumor growth in a xenograft mouse model. PSAT1 was identified as a direct target of miR-340 and its ectopic expression partially reversed the miR-340 mediated inhibition of viability, invasion and EMT in ESCC cells. The expression of miR-340 was negatively correlated with that of PSAT1 in human ESCC samples. Conclusion: MiR-340 functions as a tumor suppressor by modulating the expression of PSAT1 and may contribute to the progression and invasiveness of ESCC.

Copyright $(2015$ S. Karger AG, Basel
\end{abstract}

S. Yan, H. Jiang and S. Fang contributed equally to this work and shoud be regarded as joint first authors.

Aiwu Mao

and Tinghui Jiang

KARGER 125
Department of Interventional Radiology, Tongren Hospital, Shanghai Jiaotong University School of Medicine, No.786,Yuyuan Road, Shanghai 200050 (China) and Department of Oncology, Tongren Hospital, Shanghai Jiaotong University School of Medicine, Shanghai, 200050, (China); E-Mail aiwu_mao@126.com and E-Mail Jiangtinghui@sina.com 


\section{Introduction}

Esophageal cancer, which includes esophageal squamous cell carcinoma (ESCC) and adenocarcinoma of the esophagus, is the eighth most common cancer worldwide and the sixth most common cause of cancer-related death [1,2]. ESCC accounts for $60-70 \%$ of all cases of esophageal cancer in the world and it is more common in the developing world, occurring at a rate 20 to 30 times higher in China than in the United States [3, 4]. ESCC arises from the epithelial cells lining the esophagus and its incidence is associated with tobacco smoking, alcohol consumption, hot drinks and a poor diet [1]. ESCC is an aggressive cancer that is often diagnosed at later stages of the disease, which may explain the high mortality associated with this malignancy.

MicroRNAs (miRNAs) are small (18-25 nucleotides) noncoding RNAs that negatively regulate gene expression via base pairing to complementary sequences in the $3^{\prime}$ untranslated region (3'-UTR) of target mRNAs [5]. Approximately 1000 miRNAs have been identified in the human genome, and up to $30 \%$ of genes are thought to be regulated by miRNAs [6]. Aberrant expression of miRNAs is associated with several diseases including cancer, and tumor-associated miRNAs can function as tumor suppressors or oncogenes depending on their target mRNAs [7-11]. MiR-340 is an extensively studied miRNA that plays a tumor suppressor role in several cancers including breast, colorectal and gastric cancers [1214]. MiR-340 was shown to suppress tumor growth in osteosarcoma via targeting the rho-associated protein kinase 1 (ROCK1, a serine/threonine protein kinase involved in the regulation of the actin cytoskeleton [15]. In ESCC, several aberrantly expressed miRNAs have been identified in microarray or qRT-PCR analyses, including miR-340 [16, 17]; however, the role of miR-340 in ESCC has not been investigated to date.

Cancer cells need to alter metabolic pathways to support rapid cell growth and proliferation, and metabolic processes involving the synthesis of serine and glycine are essential to meet these requirements [18]. Several enzymes involved in one-carbon metabolism, which is a system of biological reactions that facilitates the cycling of onecarbon units for the biosynthesis of cellular components and the maintenance of redox status, are associated with tumorigenesis $[19,20]$. Among these enzymes, phosphoglycerate dehydrogenase (PHGDH), and phosphoserine aminotransferase 1 (PSAT1), which catalyze the biosynthesis of serine, are upregulated in several cancers and are suggested to contribute to the pathogenesis of human cancer by diverting the glucose flux towards serine metabolism [20]. However, the mechanisms regulating the expression of these enzymes and the contribution of alterations in metabolic pathways to tumor cell proliferation and growth are not entirely clear.

In the present study, we explored the expression and function of miR-340 in vitro and in vivo and examined the mechanisms underlying its activity. We showed that miR-340 is downregulated in ESCC tumor tissues and cell lines and identified PSAT1 as its direct target. Our results suggest that miR-340 acts as a tumor suppressor in ESCC, and its effects on the progression and invasiveness of ESCC are mediated by the downregulation of PSAT1, suggesting potential novel biomarkers and therapeutic targets for the treatment of ESCC.

\section{Materials and Methods}

\section{Patients and Tissue Specimens}

A total of 64 patients diagnosed with ESCC between 2004 and 2007 at the Tongren Hospital, who underwent esophageal cancer resection prior to the administration of chemotherapy were included in the study. ESCC and carcinoma-adjacent tissues were collected immediately after surgical resection, and confirmed by experienced pathologists. Tissues were dissected manually to remove connective tissue and stored in liquid nitrogen immediately until use. All samples were obtained from the Tissue Bank of Tongren Hospital and coded anonymously in accordance with local ethical guidelines (as stipulated by the Declaration of Helsinki). Written informed consent was obtained from all patients, and the protocol was 


\begin{tabular}{|c|c|c|}
\hline Cellular Physioloay & Cell Physiol Biochem 2015;37:375-386 & \\
\hline and Biochemistry & $\begin{array}{l}\text { DOI: } 10.1159 / 000430361 \\
\text { Publisned onnine: August 27, } 2015\end{array}$ & $\begin{array}{l}\text { (c) } 2015 \text { S. Karger AG, Basel } \\
\text { www.karger.com/cpb }\end{array}$ \\
\hline
\end{tabular}

Table 1. The primer sequences

\begin{tabular}{lll}
\hline Gene name & $\mathrm{F}\left(5^{\prime}-3^{\prime}\right)$ & $\mathrm{R}\left(5^{\prime}-3^{\prime}\right)$ \\
\hline PSAT1 & GGCCAGTTCAGTGCTGTCC & GCTCCTGTCACCACATAGTCA \\
GAPDH & CTGGGCTACACTGAGCACC & AAGTGGTCGTTGAGGGCAATG \\
\hline
\end{tabular}

approved by the Review Board of Tongren Hospital (approval number: 20120614-XF). ESCC specimens were staged in accordance with American Joint Cancer Committee/Union Internationale Contre le Cancer (UICC/AJCC) classification guidelines. The grading and histopathology subtyping of ESCC specimens was based on WHO criteria.

\section{Cell Lines and Culture Conditions}

The immortalized human esophageal endothelial cell line HEEC was purchased from Sciencell Research Laboratories (Sciencell, Carlsbad, CA, USA) and grown in Epithelial Cell Medium-2 (Sciencell) supplemented with epithelial cell growth supplement-2 (ScienCell). Human embryonic kidney HEK293T cells and three ESCC cell lines (EC1, EC109, and EC9706) were purchased from the Chinese Academy of Medical Science (Shanghai, China) and are routinely maintained in our lab in DMEM or RPMI 1640 medium (GIBCO, Carlsbad, CA) supplemented with 10\% fetal bovine serum, 100 units/ml penicillin G, and $100 \mu \mathrm{g} /$ $\mathrm{ml}$ streptomycin at $37^{\circ} \mathrm{C}$ in humidified air containing $5 \% \mathrm{CO}_{2}$.

\section{Quantitative Real-Time PCR}

Total cellular RNA was isolated from cells using Trizol (Invitrogen, Carlsbad, CA, USA) according to the manufacturer's protocol. RNA was eluted with RNase-free water, quantified by measuring absorbance at $260 / 280 \mathrm{~nm}$, and used for reverse transcription reactions.

The expression of miR-340 was measured by TaqMan assay (Applied Biosystems, Foster City, CA). MiRNA and gene expression were normalized using endogenous U6.

For PSAT1, cDNA was synthesized by oligo(dT) priming using the RevertAid ${ }^{\mathrm{TM}}$ First Strand cDNA Synthesis Kit (Fermentas, Canada) according to the manufacturer's instructions. Real-time quantitative PCR was performed with a commercial SYBR-Green master mix kit (Takara) using an Applied Biosystems Prism 7900 Sequence Detection System. GAPDH was used as an internal control. Each sample was measured in triplicate. The primer sequences are shown in Table 1.

\section{Plasmid constructs and lentiviral transduction}

To generate the miR-340 overexpressing lentiviral vector pCDH-miR-340, a fragment encoding the pre-miR-340 sequence was amplified by PCR from human genomic DNA and then cloned into the BamHI/ EcoRI sites of the pCDH-CMV-EF1-copGFP vector (SBI, Mountain View, CA, USA) with the following primers: forward, 5'-AAA GAA TTC ATC AAG ATC ACG TTT AAT ATG TGC-3'; reverse, 5' ${ }^{\prime}$-AAA GGA TCC GGT TCA CTC TTC TTC CAC ACGTA- $3^{\prime}$. Constructs and insertions were confirmed by DNA sequencing.

The pcDNA3 vector was used to generate a PSAT1 overexpression plasmid. The full-length human PSAT1 cDNA sequence was amplified by PCR using cDNA isolated from HL60 cells in which PSAT1 expression is rich as the template, and the primers used were as follows: forward, 5'- AAA GAA TTC ATG GAC GCC CCC AGG CAG-3'; reverse, 5' - AAA GGA TCC TCA TAG CTG ATG CAT CTC CAA AAAT-3'. Then the PSAT1 cDNA gene was cloned into the BamHI/EcoRI restriction sites.

The $3^{\prime}$-UTR of PSAT1 containing an intact miR-340 recognition sequence was amplified by PCR from genomic DNA using the primers 5' ${ }^{\prime}$-CCC CCT CGA GAC ACA TCC TAA CCA GGA TAT ACTCT- $3^{\prime}$ and $5^{\prime}$-AAA AGC GGC CGC GGG GAA AGA CTT TTC AGC AAAG- $3^{\prime}$. Then the PCR products were subcloned into a psiCHECK-2 ${ }^{\mathrm{TM}}$ Vector (Promega, Madison, WI). A psiCHECK-2 ${ }^{\mathrm{TM}}$ construct containing the PSAT1 $3^{\prime}$-UTR with mutations in the seed sequence was synthesized with a QuikChange Site-Directed Mutagenesis Kit (Stratagene; Agilent Technologies, Palo Alto, CA).

For production of viral particles, the lentivirus-mediated PSAT1 packaging system containing pCDH-CMV-EF1-copGFP or pCDH-miR-340, Rec, TAT, Gag, and Vsvg was transfected into HEK293T cells using Lipofectamine ${ }^{\mathrm{TM}} 2000$ (Invitrogen) according to the manufacturer's instructions. The supernatant containing the virus source was collected $60 \mathrm{~h}$ post-transfection and filtered through a $0.45-\mu \mathrm{m}$ pore membrane (Millipore). 
Cells were grown to log phase and then transfected with either pCDH-CMV-EF1-copGFP (vector) or pCDH-miR-340 for $24 \mathrm{~h}$. Stable cell lines were screened by mass sorting on a FACSAria flow cytometer (BD Biosciences, Mountain View, CA, USA) based on the expression of GFP carried by the lentiviral vector $72 \mathrm{~h}$ after transfection.

Gene transfection

ESCC cells $\left(5 \times 10^{6}\right)$ in $2 \mathrm{ml}$ of culture medium were transfected with 100 pmol of control vector or PSAT1 cDNA mixed with Lipofectamine 2000 reagent in serum reduced medium according to the manufacturer's instructions. The medium was changed to complete culture medium after $6 \mathrm{~h}$, and the cells were incubated at $37^{\circ} \mathrm{C}$ in a $\mathrm{CO}_{2}$ incubator for an additional $48 \mathrm{~h}$ before harvest.

\section{Luciferase reporter assays}

HEK-293T cells were cotransfected with $50 \mathrm{nM}$ of either miR-340 mimic or the negative control and $500 \mathrm{ng}$ of psiCHECK2-PSAT1-3'-UTR-wt or psiCHECK-2- PSAT1-3'-UTR-mut. Cells were collected $48 \mathrm{~h}$ after transfection and analyzed using the Dual-Luciferase Reporter Assay System (Promega, San Luis Obispo, CA). Luciferase activity was detected using a GloMax fluorescence reader (Promega, San Luis Obispo, CA). A psiCHECK-2 vector that provides constitutive expression of Renilla luciferase was cotransfected as an internal control.

\section{Invasion assays}

For cell invasion assays, an $8 \mu \mathrm{m}$ pore filter membrane (Corning, Acton, MA) was coated with Matrigel (diluted at 1:5) (BD Biosciences) in serum-free RPMI 1640. After air-drying and sterilization, $29 \mu \mathrm{l}$ of the plain medium with serum was added to the lower chamber of the Costar Transwell (Corning). Cells transfected as indicated were trypsinized, resuspended in serum-free RPMI 1640 at a density of $1.2 \times 10^{6}$ cells $/ \mathrm{ml}$, seeded in $50 \mu \mathrm{l}$ in the upper chamber, and incubated at $37^{\circ} \mathrm{C}$ for $20 \mathrm{~h}$. Non-invading cells remaining on the upper surface of the filter were removed with a cotton swab and rinsed with PBS several times; cells that appeared on the lower surface of the filter were fixed with methanol for more than $15 \mathrm{~min}$ at $-20^{\circ} \mathrm{C}$ and then washed in PBS, stained with crystal violet and counted under a microscope.

\section{Proliferation assay}

To measure cell viability, cells treated as indicated were seeded into 96-well plates at a density of $4 \times 10^{3}$ cells/well $/ 200 \mu \mathrm{L}$ in maintenance medium. Viable cells were quantified at $24,48,72$ and $96 \mathrm{~h}$ after the seeding by the chromogenic 3-[4, 5-dimethylthiazol-2-yl]-2,5-diphenyl-tetrazolium-bromide (MTT) assay according to the manufacturer's instructions (Sigma, St. Louis, US). Each assay was performed in triplicate and repeated three times.

\section{Colony formation assay}

Colony formation assays were performed using a previously described method [13]. Briefly, cells were digested with trypsin and suspended into a single cell status. Approximately 6000 cells from each group were cultured in a $60 \mathrm{~mm}$ diameter culture dish with $10 \%$ FBS for 14 days. The colonies were fixed and stained with $0.5 \%$ crystal violet for $15 \mathrm{~min}$, and then washed three times. Colonies consisting of more than 50 cells were defined as one colony. The number of colonies in 10 random view fields was counted under a microscope and the average representing the $95 \%$ confidence region was achieved. The experiment was performed three independent times.

\section{Tumorigenicity in BALB/c Scid Mice}

Animal experiments were performed according to the Institutional Animal Care and Use Committee guidelines of the Experiment Animal center of Shanghai Jiaotong University School of Medicine, and the approval ID was No 140811-J by Experiment Animal Center of Shanghai Jiaotong University School of Medicine. BALB/C-nu/nu nude mice were obtained from Shanghai Laboratory Animal Center of China and housed under specific pathogen-free conditions. Approximately $1 \times 10^{7}$ cells of each cell line in $200 \mu \mathrm{l}$ of medium without serum were injected subcutaneously into the upper back of nude mice. Tumor growth was measured with calipers every 7 days, and tumor volumes were calculated using the following formula: $1 / 2$ (length $\times w_{\text {width }}^{2}$ ). Mice were sacrificed and tumor weight was examined 5 weeks later. 
Western blotting

Cells treated as indicated were lysed in RIPA lysis buffer (Cell Signaling Technology, Beverly, MA, USA) and equal amounts of protein were separated by sodium dodecyl sulfate polyacrylatmide gel electrophoresis and transferred onto PVDF membranes (Millipore, Boston, MA). Membranes were blocked with $5 \%$ skim milk in Tris buffered saline containing $0.1 \%$ Tween 20 (TBS-T), followed by incubation with primary antibodies at $4^{\circ} \mathrm{C}$ overnight. The following antibodies were used: p-GSK-3 $\beta$, GSK-3 $\beta$, Snail, E-cadherin, Vimentin (Cell Signaling Technology); and PSAT1 (Proteintech Group Inc, Chicago, IL). Anti-GAPDH antibody (Santa Cruz Biotechnology) was used as an internal control. After incubation with the appropriate peroxidaseconjugated secondary antibodies for $2 \mathrm{~h}$, blots were visualized by enhanced chemiluminescence.

\section{Statistical analysis}

All data were presented as mean \pm SD. Differences were analyzed by t-test or analysis of variance. Differences with a p value $<0.05$ were considered statistically significantly. For western blotting, the relative protein levels were quantified by the ratio to the loading control GAPDH, and the mean \pm S.D. of three blots is shown.

\section{Results}

MiR-340 is downregulated in ESCC cell lines and tissues

Carcinoma and adjacent non-tumor tissues from 64 ESCC patients were analyzed by qRT-PCR to compare the levels of miR-340 expression. The results showed that miR-340 expression was significantly lower in tumor tissues than in adjacent non-tumor tissues $(\mathrm{p}<$ 0.01) (Fig. 1A). MiR-340 was significantly downregulated in the ESCC cell lines EC1, EC109, and EC9706 compared to the human esophageal endothelial cell line HEEC, with the lowest levels of miR-340 expression observed in EC9706 cells followed by EC109 cells (Fig. 1B). Therefore, these two cell lines were used for subsequent experiments.

MiR-340 suppresses ESCC cell growth in vitro and in vivo

To investigate the role of miR-340 in ESCC, cells were transfected with a miR-340 overexpression vector and cell viability and tumor growth were measured. Overexpression of miR-340 in the EC109 and EC9706 cell lines was confirmed by qRT-PCR (Fig. 2A). MiR340 overexpression significantly inhibited cell viability and colony formation in EC109 and EC9706 cells as determined by the MTT and colony forming assays (Fig. 2B and C). Assessment of tumor growth in a mouse xenograft model showed that tumor weight was significantly lower in mice injected with miR-340 overexpressing EC9706 cells than in those injected with vector control cells ( $\mathrm{p}<0.021$ ) (Fig. 2D).

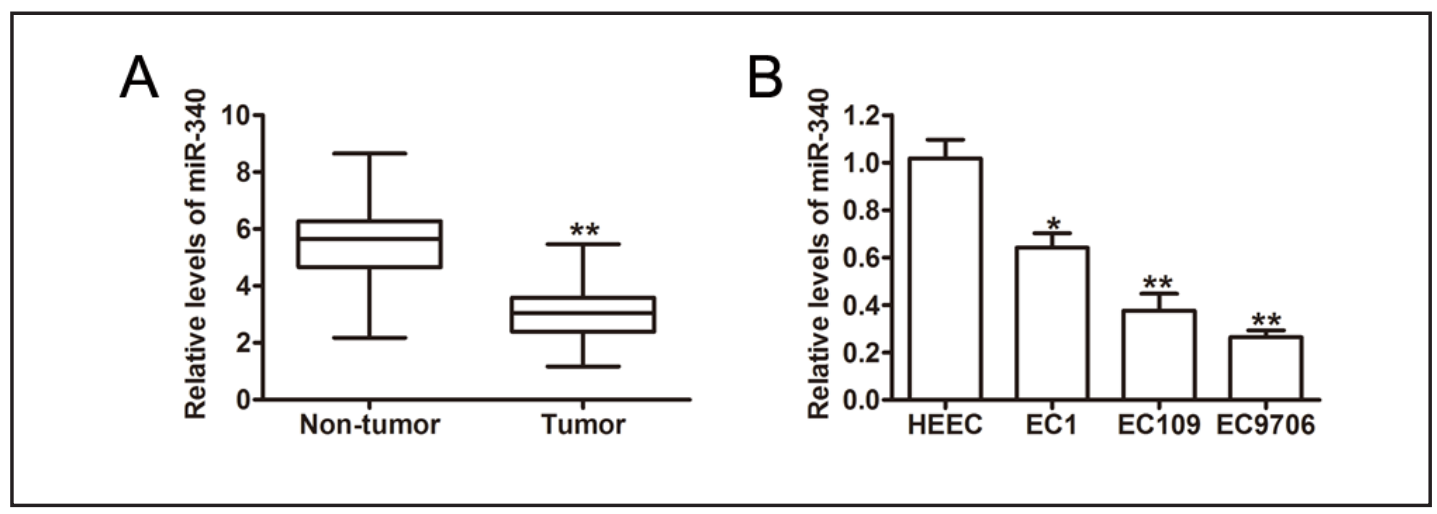

Fig. 1. miR-340 is downregulated in human ESCC. (A) qRT-PCR analysis of miR-340 levels in tumor and adjacent non-tumor tissues of 64 patients with ESCC. ${ }^{* *}, \mathrm{p}<0.01$. (B) qRT-PCR analysis of miR-340 expression in ESCC cell lines and human esophageal endothelial cells. 


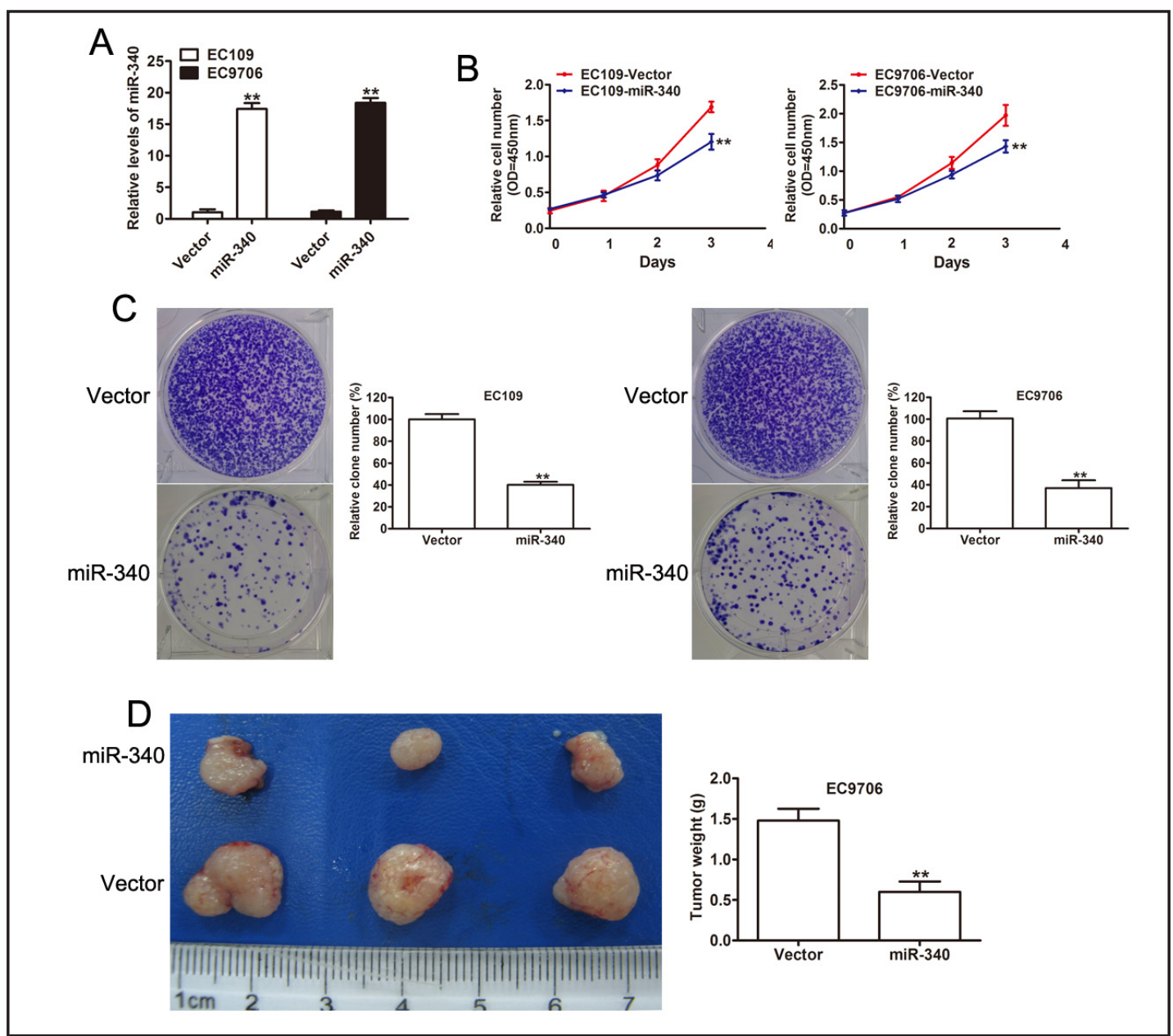

Fig. 2. Effect of miR-340 on cell growth in vitro and in vivo. (A) miR-340 expression in stable transfectants of EC109 and EC7906 cells was detected by qRT-PCR. Results are representative of three independent experiments. (B) Cell viability of EC109 and EC7906 stable transfectants measured by the MTT assay. Data represent the mean \pm standard deviation of three experiments. (C) Colony formation was assayed in miR340 and vector-infected EC109 and EC7906 cells; colonies consisting of more than 50 cells were counted. The experiment was repeated three times independently. (D) Mice were injected with EC7906-Vector or EC7906-miR-340 infected cells and nursed for 35 days. Tumor weight was measured. Data represent the mean \pm standard deviation (Student's t-test, $p=0.021$ ).

\section{MiR-340 suppresses invasion in ESCC cell lines}

To further explore the effect of miR-340 on ESCC, cells transfected with or without a miR340 overexpressing vector were analyzed by Matrigel invasion assays. The results showed that miR-340 overexpression significantly inhibited the invasion of EC109 and EC9706 cells by approximately $60 \%$ of the control $(\mathrm{p}<0.01$ ) (Fig. $3 \mathrm{~A}$ and $\mathrm{B}$ ).

PSAT1 is a direct target gene of $\mathrm{miR}-340$

To further explore the regulating mechanisms of miR-340, we examined its potential targets using prediction tools. A conserved domain within the 3 '-UTR of PSAT1 with two potential miR-340 binding sites was identified using the prediction algorithm of Targetscan (Fig. 4A). To confirm this prediction, luciferase assay was performed to validate PSAT1 as a target of miR-340. HEK293 cells were co-transfected with luciferase reporter constructs generated by cloning the wild-type or mutant $3^{\prime}$-UTR of PSAT1 carrying mutations in the miR-340 seed region and the miR-340 overexpressing vector or control vector. Assessment of 
Fig. 3. The effect of PSAT1 on cell invasion in vitro. (A) Representative images of basement membrane matrix invasion assays in EC109 cells transduced with miR-340-expressing or control vector. (B) Representative images of basement membrane matrix invasion assay in EC9706 cells transfected with miR-340-expressing or control vector. Quantitative analysis shows the relative number of invaded cells counted microscopically in five different fields per filter. Data represent the mean \pm standard deviation of three experiments.
Fig. 4. PSAT1 is a direct target of miR-340. (A) The putative miR-340-binding sequence in the $3^{\prime}$-UTR of PSAT1 mRNA is shown. Mutations were introduced into the PSAT1 3'-UTR sequence at the complementary site for the seed region of miR-340. (B) Luciferase reporter assays show the miR-340-mediated suppression of the activity of the wild-type PSAT1 3'-UTR luciferase, but not the mutant 3 '-UTR luciferase, in HEK-293 cells. (C) Ectopic expression of miR-340 in EC109 and EC7906 cells downregulated the mRNA expression of PSAT1. (D) PSAT1, GSK$3 \beta$, p-GSK-3 $\beta$ (Ser9), Snail, Vimentin and E-cadherin protein levels were determined by western blotting. *, $\mathrm{p}<0.05 ; * *, \mathrm{p}<0.01$.

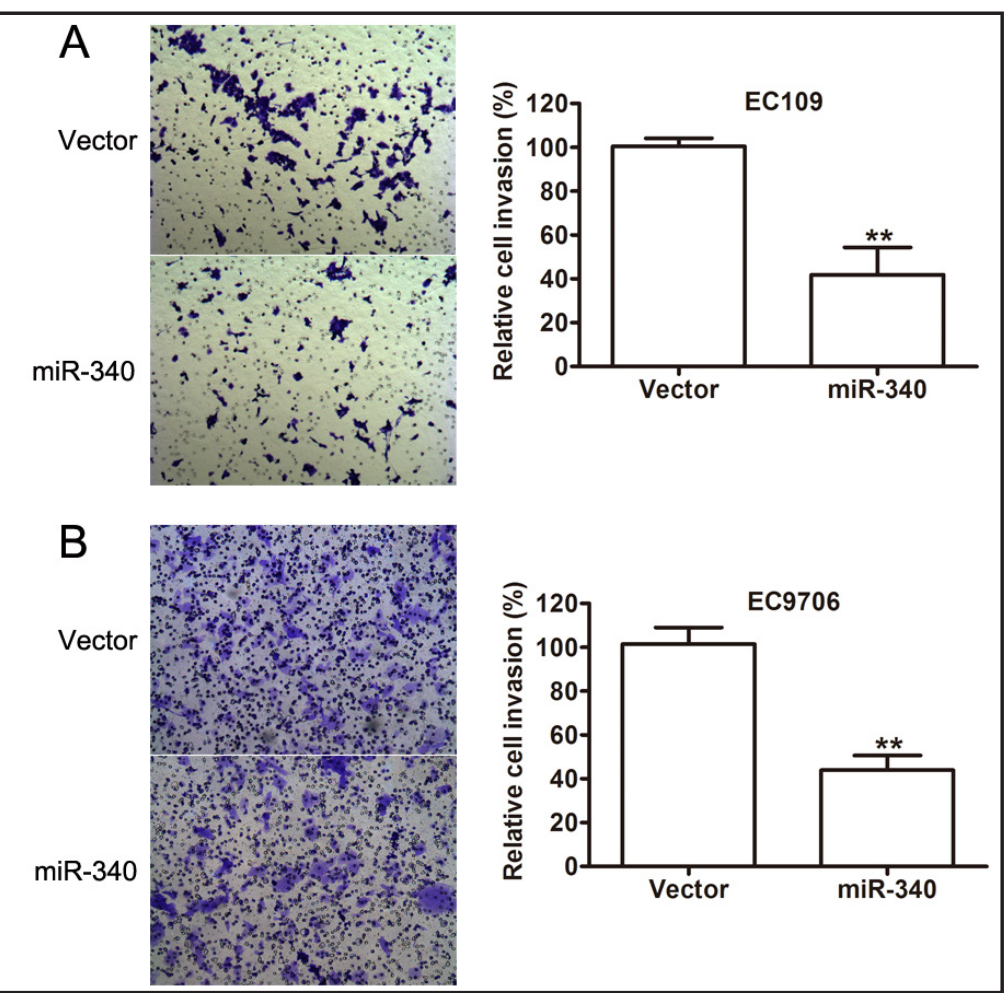

A 534-540 of PSAT1 3' UTR 5'...AGGAGAAGUGACAUACUUUAUAU...3' || || | | hsa-miR-340 3' UUAGUCAGAGUAACGAAAUAUU 5' | | || | | | 580-586 of PSAT1 3' UTR $\quad 5^{\prime}$...UUUUAAUACCAUAUACUUUAUAU...3'
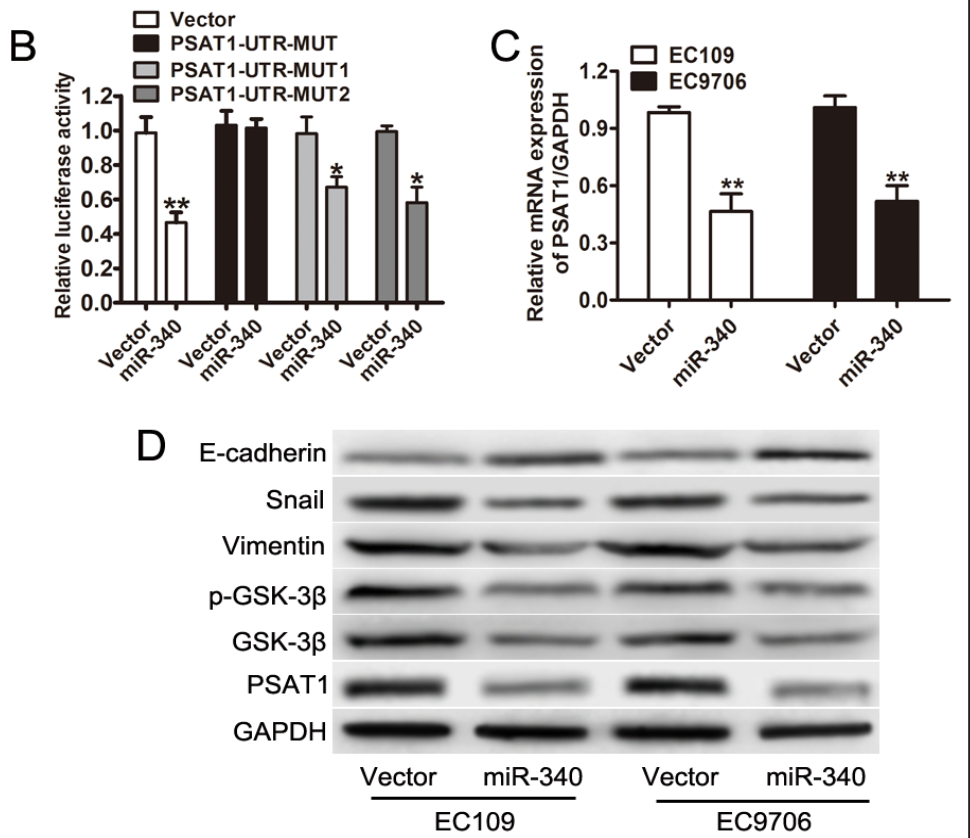

luciferase activity showed that miR-340 overexpression significantly decreased the reporter activity of the wild-type, PSAT1-UTR-MUT1 or PSAT1-UTR-MUT2, but not that of the two KARGER 
Fig. 5. Ectopic expression of PSAT1 rescues the biological effects of miR-340 overexpression. (A) The growth rate of EC109 and EC7906 cells stably overexpressing miR-340 and transfected with PSAT1 was measured with the MTT assay. (B) Transwell assays were performed in EC109 and EC7906 cells stably overexpressing miR-340 and transfected with PSAT1. (C) PSAT1 transfection abrogated the miR-340-induced upregulation of GSK-3 $\beta$, p-GSK-3 $\beta$ (Ser9), Snail and Vimentin, and miR-340-induced downregulation of E-cadherin. *, p < 0.05; **, p $<0.01$.
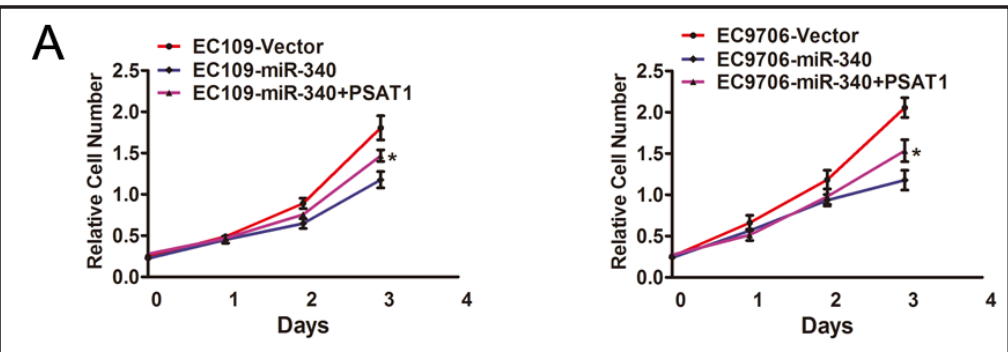

B
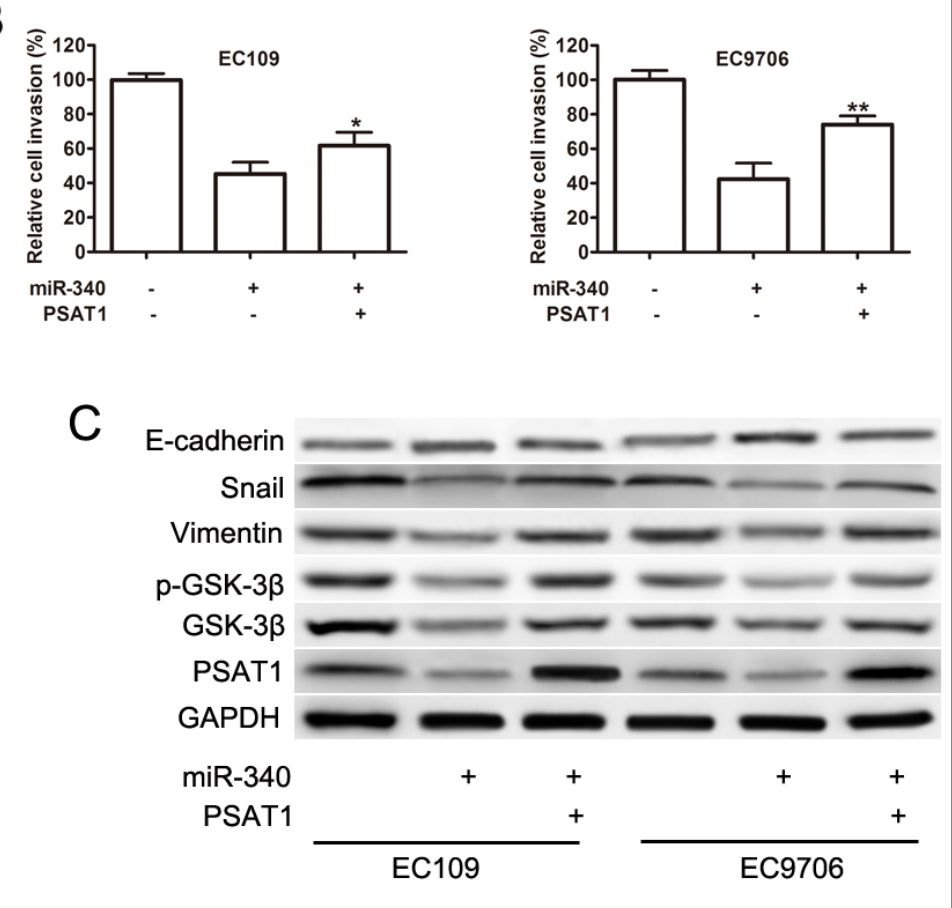

binding sites mutations of PSAT 3'-UTR (Fig. 4B). Ectopic expression of miR-340 significantly downregulated the mRNA expression of PSAT1 relative to that of the GAPDH control in both EC109 and EC9706 cells (Fig. 4C), further confirming that PSAT1 is a target of miR-340. The effect of miR-340 on tumor invasiveness was further examined by western blot analysis of the expression of the epithelial marker E-cadherin, the epithelial-mesenchymal transition (EMT) markers Snail and Vimentin, and the Ser/Thr kinase GSK-3 $\beta$. The transcription factor Snail regulates the expression of E-cadherin, and downregulation of E-cadherin induces EMT [21]. GSK-3 $\beta$ is a Ser/Thr kinase that is inactivated by phosphorylation at Ser9; it phosphorylates and regulates Snail activity, and it maintains the epithelial phenotype by inhibiting Snail, and inactivation of GSK-3 $\beta$ promotes EMT. As shown in Fig. 4D, overexpression of miR340 upregulated E-cadherin, downregulated Snail and Vimentin, and reduced the levels of phosphorylated and total GSK-3 $\beta$ concomitant with the downregulation of PSAT1, indicating that miR-340 might suppresse EMT in correlation with the downregulation of its target PSAT1 in ESCC cells.

PSAT1 is involved in miR-340-induced suppression of ESCC cell proliferation and invasion

To confirm the relationship between the miR-340/PSAT1 axis and the regulation of ESCC cell proliferation and invasion, PSAT1 was ectopically expressed in ESCC cells and cell viability and invasiveness were assessed. The results showed that ectopic expression of PSAT1 partially rescued the inhibition of cell proliferation and invasion induced by miR340 overexpression in both EC109 and EC9706 cells (Fig. 5A and B). Consistently, ectopic expression of PSAT1 partially restored the levels of E-cadherin and Snail/Vimentin down- 
Fig. 6. PSAT1 expression is inversely correlated with miR-340 expression in ESCC. Correlation analysis of PSAT1 and miR-340 expression in samples from 64 patients with ESCC $\left(R^{2}=0.3073 ; p<0.01\right)$.

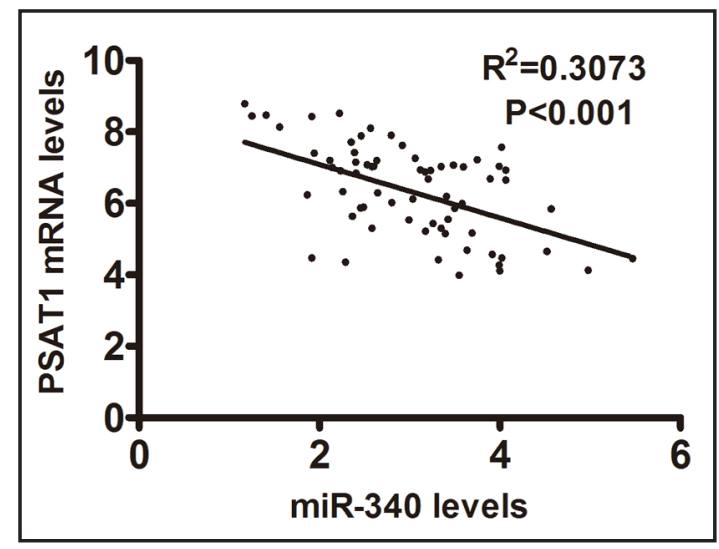

regulated by miR-340, respectively, suggesting that PSAT1 reverses the effect of miR-340 on suppressing EMT in ESCC cells (Fig. 5C). qRT-PCR analysis of miR-340 and PSAT1 expression in samples from patients with ESCC showed a negative correlation between the mRNA levels of PSAT1 and the expression of miR-340 $\left(\mathrm{r}^{2}=0.3073, \mathrm{p}<0.01\right)$, further confirming that miR340 regulates the expression of PSAT1 in ESCC (Fig. 6).

\section{Discussion}

In the present study, we explored the expression and role of miR-340 in ESCC and the mechanisms underlying its activity. We show that miR-340 is downregulated in ESCC cell lines and tissues and its overexpression inhibits cell viability, colony formation, and invasion in vitro and tumor growth in a mouse xenograft model in vivo. We identified PSAT1 as a direct target of miR-340 and showed that its expression is negatively correlated with that of miR340 in ESCC tumor samples. Our results suggest that miR-340 acts as a tumor suppressor by modulating the expression of PSAT1, and its downregulation may contribute to the progression and invasiveness of ESCC.

Esophageal cancer is an aggressive malignancy associated with a poor prognosis, and the 5-year survival rate of ESCC patients is approximately 19\% [22, 23]. The high mortality rate of ESCC can be partly attributed to the fact that it is typically diagnosed at an advanced stage, underscoring the need to identify biomarkers for the early detection of this disease. Several studies have focused on identifying miRNAs associated with esophageal cancer using microarray or expression analyses. Guo et al. reported the results of a miRNA array containing 509 miRNA sequences in which miRNA expression in 31 matched ESCC and normal tissues was compared [24]. This study identified 7 miRNAs capable of distinguishing ESCC from normal tissues, among which three (miR-25, miR-424, and miR-151) were upregulated and four (miR-100, miR-99a, miR-29c and miR-140) were downregulated in ESCC. In a microarray analysis of 210 human miRNAs, four miRNAs, let-7d, miR-330, miR340, and miR-373, were upregulated in ESCC tumors [16]. Ogawa et al. analyzed 73 miRNAs by qRT-PCR and identified six upregulated miRNAs and four downregulated miRNAs in ESCC tissues compared to paired normal tissues, whereas Kano et al. used Taqman human miRNA array to examine 365 human miRNAs and found 15 downregulated miRNAs in ESCC tissues $[25,26]$. Only a few miRNAs overlap among these studies, indicating that the involvement of miRNAs in ESCC is complex and requires further investigation. Among the miRNAs consistently shown to act as tumor suppressors in ESCC, miR-203, miR-205, miR-10a, and miR375 have been shown to be downregulated in ESCC compared to non-tumor tissues [16, 27]. We showed that miR-340 is downregulated in tumor tissues compared to adjacent nontumor tissues and in ESCC cell lines, and its expression is associated with the viability, colony formation ability and invasiveness of ESCC cell lines and tumor growth in a mouse model.

Although several studies have examined the role of miRNAs in esophageal cancer, few potential targets have been identified. Three miRNAs (miR-145, miR-133a, and miR-133b) 
inhibit cell proliferation and invasion when transfected into ESCC cells and all three have conserved sequences in the 3'-UTR of FSCN1 (actin-binding protein, Fascin homolog 1) and inhibit its expression [26]. miR-133a was shown to act as a tumor suppressor in ESCC by targeting CD47 [28], and miR-25 was shown to interact with and inhibit the activity of CDH1, a known tumor suppressor that encodes E-cadherin, suggesting that miR-25 promotes ESCC cell migration and invasion by downregulating CDH1 [29]. Other miRNAs and their targets involved in ESCC include miR-518b and Rap1b, miR-196a and Rap1a, miR-223 and FBXW7, miR-150 and ZEB1, and miR-143 and ERK5 [30]. Here, we showed that miR-340 acts as a tumor suppressor in ESCC by targeting PSAT1. Overexpression of miR-340 downregulated PSAT1, and ectopic expression of PSAT1 reversed the miR-340 induced inhibition of cell proliferation, invasion and EMT. Alterations in the expression of enzymes of the serine biosynthesis pathway, including PHGDH and PSAT1, have been associated with several cancers. In breast cancer, an RNAi based loss of function screening revealed that overexpression of enzymes involved in the serine biosynthesis pathway is required for in vivo tumorigenesis [31]. PHGDH was shown to be upregulated in certain breast cancers, and suppression of PSAT1 inhibited the proliferation of PHGDH overexpressing breast cancer cell lines [32, 33]. PSAT1 is overexpressed in colorectal cancer and is associated with chemoresistance, and increased levels of PSAT1 mRNA have been detected in colon adenocarcinoma, and correlated with tumor stage in colon cancer [31]. In breast cancer, PSAT1 is associated with poor responses to endocrine therapy [33]. These studies demonstrate the involvement of PSAT1 in tumorigenesis and support our present results suggesting that the downregulation of miR-340 may contribute to tumorigenesis in ESCC via the upregulation of PSAT1. Further studies focusing on the relation between miR-340 and PSAT1 may clarify their roles in ESCC development and progression.

\section{Conclusions}

The results of the present study indicate that miR-340 acts as a tumor suppressor in ESCC via the modulation of the expression of its target PSAT1, a serine biosynthesis pathway enzyme associated with tumorigenesis in several cancers. Our results showing that the effects of miR-340 on cell proliferation and invasion are reversed by the ectopic expression of PSAT1 and that the expression of these proteins is negatively correlated in ESCC samples confirm that miR-340 exerts its effects through PSAT1 and suggest potential novel biomarkers in ESCC.

\section{Disclosure Statement}

The authors have no conflicts of interest.

\section{References}

1 Arnold M, Soerjomataram I, Ferlay J, Forman D: Global incidence of oesophageal cancer by histological subtype in 2012. Gut 2015;64:381-387.

-2 Lagergren J: Oesophageal cancer in 2014: Advances in curatively intended treatment. Nat Rev Gastroenterol Hepatol 2015;12:74-75.

3 Zhang Y: Epidemiology of esophageal cancer. World J Gastroenterol 2013;19:5598-5606.

-4 Liu P, Gao Y, Huan J, Ge X, Tang Y, Shen W, Tian Y, Shen W, Zou S, Zhou J, Zhang S: Upregulation of PAX2 promotes the metastasis of esophageal cancer through interleukin-5. Cell Physiol Biochem 2015;35:740754.

-5 Shukla GC, Singh J, Barik S: MicroRNAs: Processing, maturation, target recognition and regulatory functions. Mol Cell Pharmacol 2011;3:83-92. 
6 Nana-Sinkam SP, Croce CM: Clinical applications for microRNAs in cancer. Clin Pharmacol Ther 2013;93:98-104.

7 Osman A: MicroRNAs in health and disease--basic science and clinical applications. Clin Lab 2012;58:393402.

-8 Esquela-Kerscher A, Slack FJ: Oncomirs - microRNAs with a role in cancer. Nat Rev Cancer 2006;6:259-269.

-9 Wang QX, Zhu YQ Zhang H, Xiao J: Altered MiRNA expression in gastric cancer: A systematic review and meta-analysis. Cell Physiol Biochem 2015;35:933-944.

10 Li W, Jiang G, Zhou J, Wang H, Gong Z, Zhang Z, Min K, Zhu H, Tan Y: Down-regulation of miR-140 induces EMT and promotes invasion by targeting Slug in esophageal cancer. Cell Physiol Biochem 2014;34:14661476.

11 Zhu Y, Xia Y, Niu H, Chen Y: MiR-16 induced the suppression of cell apoptosis while promote proliferation in esophageal squamous cell carcinoma. Cell Physiol Biochem 2014;33:1340-1348.

12 Wu ZS, Wu Q, Wang CQ Wang XN, Huang J, Zhao JJ, Mao SS, Zhang GH, Xu XC, Zhang N: MiR-340 inhibition of breast cancer cell migration and invasion through targeting of oncoprotein c-Met. Cancer 2011;117:2842-2852.

-13 Fernandez S, Risolino M, Mandia N, Talotta F, Soini Y, Incoronato M, Condorelli G, Banfi S, Verde P: MiR-340 inhibits tumor cell proliferation and induces apoptosis by targeting multiple negative regulators of p27 in non-small cell lung cancer. Oncogene 2015;34:3240-3250

14 Huang D, Qiu S, Ge R, He L, Li M, Li Y, Peng Y: MiR-340 suppresses glioblastoma multiforme. Oncotarget 2015;6:9257-9270

15 Zhou X, Wei M, Wang W: MicroRNA-340 suppresses osteosarcoma tumor growth and metastasis by directly targeting ROCK1. Biochem Biophys Res Commun 2013;437:653-658.

16 Lee KH, Goan YG, Hsiao M, Lee CH, Jian SH, Lin JT, Chen YL, Lu PJ: MicroRNA-373 (miR-373) posttranscriptionally regulates large tumor suppressor, homolog 2 (LATS2) and stimulates proliferation in human esophageal cancer. Exp Cell Res 2009;315:2529-2538.

17 Zhang M, Yang Q, Zhang L, Zhou S, Ye W, Yao Q, Li Z, Huang C, Wen Q, Wang J: MiR-302b is a potential molecular marker of esophageal squamous cell carcinoma and functions as a tumor suppressor by targeting ErbB4. J Exp Clin Cancer Res 2014;33:10.

18 Ma L, Tao Y, Duran A, Llado V, Galvez A, Barger JF, Castilla EA, Chen J, Yajima T, Porollo A, Medvedovic M, Brill LM, Plas DR, Riedl SJ, Leitges M, Diaz-Meco MT, Richardson AD, Moscat J: Control of nutrient stressinduced metabolic reprogramming by PKCzeta in tumorigenesis. Cell 2013;152:599-611.

19 Chen J, Gammon MD, Chan W, Palomeque C, Wetmur JG, Kabat GC, Teitelbaum SL, Britton JA, Terry MB, Neugut AI, Santella RM: One-carbon metabolism, MTHFR polymorphisms, and risk of breast cancer. Cancer Res 2005;65:1606-1614.

20 Locasale JW, Grassian AR, Melman T, Lyssiotis CA, Mattaini KR, Bass AJ, Heffron G, Metallo CM, Muranen T, Sharfi H, Sasaki AT, Anastasiou D, Mullarky E, Vokes NI, Sasaki M, Beroukhim R, Stephanopoulos G, Ligon AH, Meyerson M, Richardson AL, Chin L, Wagner G, Asara JM, Brugge JS, Cantley LC, Vander HM: Phosphoglycerate dehydrogenase diverts glycolytic flux and contributes to oncogenesis. Nat Genet 2011;43:869-874.

-21 Kim JY, Kim YM, Yang CH, Cho SK, Lee JW, Cho M: Functional regulation of Slug/Snail2 is dependent on GSK-3beta-mediated phosphorylation. FEBS J 2012;279:2929-2939.

22 Jemal A, Siegel R, Xu J, Ward E: Cancer statistics, 2010. CA Cancer J Clin 2010;60:277-300.

-23 Li YJ, Zhou JH, Du XX, Jia DX, Wu CL, Huang P, Han Y, Sui H, Wei XL, Liu L, Yuan HH, Zhang TT, Zhang WJ, Xie R, Lang XH, Liu T, Jiang CL, Wang LY, Bai YX: Dihydroartemisinin accentuates the anti-tumor effects of photodynamic therapy via inactivation of NF-kappaB in Eca109 and Ec9706 esophageal cancer cells. Cell Physiol Biochem 2014;33:1527-1536.

24 Guo Y, Chen Z, Zhang L, Zhou F, Shi S, Feng X, Li B, Meng X, Ma X, Luo M, Shao K, Li N, Qiu B, Mitchelson $\mathrm{K}$, Cheng J, He J: Distinctive microRNA profiles relating to patient survival in esophageal squamous cell carcinoma. Cancer Res 2008;68:26-33.

25 Ogawa R, Ishiguro H, Kuwabara Y, Kimura M, Mitsui A, Katada T, Harata K, Tanaka T, Fujii Y: Expression profiling of micro-RNAs in human esophageal squamous cell carcinoma using RT-PCR. Med Mol Morphol 2009;42:102-109. 
-26 Kano M, Seki N, Kikkawa N, Fujimura L, Hoshino I, Akutsu Y, Chiyomaru T, Enokida H, Nakagawa M, Matsubara H: MiR-145, miR-133a and miR-133b: Tumor-suppressive miRNAs target FSCN1 in esophageal squamous cell carcinoma. Int J Cancer 2010;127:2804-2814.

-27 Feber A, Xi L, Luketich JD, Pennathur A, Landreneau RJ, Wu M, Swanson SJ, Godfrey TE, Litle VR: MicroRNA expression profiles of esophageal cancer. J Thorac Cardiovasc Surg 2008;135:255-260, 260.

-28 Suzuki S, Yokobori T, Tanaka N, Sakai M, Sano A, Inose T, Sohda M, Nakajima M, Miyazaki T, Kato H, Kuwano H: CD47 expression regulated by the miR-133a tumor suppressor is a novel prognostic marker in esophageal squamous cell carcinoma. Oncol Rep 2012;28:465-472.

29 Xu X, Chen Z, Zhao X, Wang J, Ding D, Wang Z, Tan F, Tan X, Zhou F, Sun J, Sun N, Gao Y, Shao K, Li N, Qiu B, He J: MicroRNA-25 promotes cell migration and invasion in esophageal squamous cell carcinoma. Biochem Biophys Res Commun 2012;421:640-645.

30 Sakai NS, Samia-Aly E, Barbera M, Fitzgerald RC: A review of the current understanding and clinical utility of miRNAs in esophageal cancer. Semin Cancer Biol 2013;23:512-521.

-31 Vie N, Copois V, Bascoul-Mollevi C, Denis V, Bec N, Robert B, Fraslon C, Conseiller E, Molina F, Larroque C, Martineau P, Del RM, Gongora C: Overexpression of phosphoserine aminotransferase PSAT1 stimulates cell growth and increases chemoresistance of colon cancer cells. Mol Cancer 2008;7:14.

-32 Pollari S, Kakonen SM, Edgren H, Wolf M, Kohonen P, Sara H, Guise T, Nees M, Kallioniemi O: Enhanced serine production by bone metastatic breast cancer cells stimulates osteoclastogenesis. Breast Cancer Res Treat 2011;125:421-430.

-33 Martens JW, Nimmrich I, Koenig T, Look MP, Harbeck N, Model F, Kluth A, Bolt-de VJ, Sieuwerts AM, Portengen H, Meijer-Van GM, Piepenbrock C, Olek A, Hofler H, Kiechle M, Klijn JG, Schmitt M, Maier S, Foekens JA: Association of DNA methylation of phosphoserine aminotransferase with response to endocrine therapy in patients with recurrent breast cancer. Cancer Res 2005;65:4101-4117. 